\title{
A Novel Detector to Detect Colluded Non-Technical Loss Frauds in Smart Grid
}

\author{
Wenlin Han, Yang Xiao*
}

\begin{abstract}
A Non-Technical Loss (NTL) fraud occurs when a fraudster tampers with a smart meter so that the meter registers less electricity consumption than the actual consumed amount, and therefore the utility becomes the victim who suffers the corresponding economic loss. In the literature, many detection schemes have been proposed to detect NTL frauds. However, some NTL frauds are far more complicated than what the existing schemes expect. We recently discovered a new potential type of frauds, a variant of NTL frauds, called Colluded Non-Technical Loss (CNTL) frauds in the Smart Grid. In a CNTL fraud, multiple fraudsters can co-exist or collaborate to commit the fraud. Existing detection schemes cannot detect CNTL frauds since these methods do not consider the co-existing or collaborating fraudsters, and therefore cannot distinguish one from many fraudsters. In this paper, we propose a CNTL fraud detector to detect CNTL frauds. The proposed method can quickly detect a tampered meter based on recursive least squares. After identifying the tampered meter, the proposed scheme can detect different fraudsters using mathematical models. Our experiments show that our method is effective in detecting CNTL frauds.
\end{abstract}

Index Terms-Smart Grid Security, Smart Meter, NonTechnical Loss, Colluded Fraud, Malicious Meter.

\section{INTRODUCTION}

Smart Grid is the new generation of power grid with two-way electricity flows and two-way communication links [1], [2]. The system losses in Smart Grid can be classified into two types [3]: technical loss and non-technical loss. Technical loss is the loss during the generation, transmission, distribution, and redistribution processes, and it is due to the loss of energy in power lines, transformers, and other devices. Generally, Non-Technical Loss (NTL) is referred as to the loss other than technical loss, including unpaid bills, electricity theft, inaccurate metering, etc. An NTL fraud occurs when a fraudster tampers with a smart meter so that the meter registers less electricity consumption than the actual consumed amount, and this causes non-technical loss to the utility [4], [5].

Utility companies have experienced huge economic loss because of NTL frauds. NTL frauds cause yearly $\$ 89.3 \mathrm{bn}$ economic loss world-wide as reported by Northeast Group LLC [6]. Among the $\$ 89.3 \mathrm{bn}$ loss, $\$ 58.7 \mathrm{bn}$ loss is reported in the 50 emerging market countries, including India, China, etc. These 50 countries planned an investment of totally $\$ 168 \mathrm{bn}$

Wenlin Han is with the Department of Computer Science, the University of Alabama, Tuscaloosa, AL 35487-0290, USA. E-mail: whan2@crimson.ua.edu.

Yang Xiao is with both School of Computer and Software, Nanjing University of Information Science and Technology, Nanjing, 210044, China, and with the Department of Computer Science, the University of Alabama, Tuscaloosa, AL 35487-0290, USA. E-mail: yangxiao@ieee.org.

Yang Xiao is the corresponding author. E-mail: yangxiao@ieee.org. to make Smart Grid more reliable and to reduce NTL frauds. In the U.S., the annual loss due to NTL frauds is $\$ 6 \mathrm{bn}$, and it is reported by law enforcement in Houston that probably $60 \%$ to $70 \%$ of meters have been tampered with [7].

NTL frauds not only exist in Smart Grid, but also in the old power grid [8]. In the traditional power grid, people have a long history of using physical methods to steal electricity [3], [9]. A typical method is to use an additional power line to bypass a meter. Smart Grid brings novel technologies to the power grid. However, when meters become electronic and smart, there are much more ways to commit an NTL fraud. Examples are given as follows: smart meters could be remotely controlled [10]; the communication between the meters and the utility could be intercepted; moreover, those physical methods can be still applied in Smart Grid.

Traditional NTL fraud detection methods attempt to identify a tampered meter or multiple tampered meters among meters [11], [12]. However, an NTL fraud can be very sophisticated. In our recent studies, we discovered a new potential type of frauds, a variant of NTL frauds, called Colluded NonTechnical Loss (CNTL) frauds in the Smart Grid. In a CNTL fraud, more than one fraudster can co-exist or collaborate to commit the fraud. In other words, in a single tampered meter, there are multiple fraudsters. They may not realize the existence of other fraudsters, and we call them co-existing fraudsters. On the other hand, they may realize the existence of other fraudsters and collaborate to commit an NTL fraud, and then we call them collaborating fraudsters. We study the behaviors of the co-existing and collaborating fraudsters and analyze the features of CNTL frauds. We classify CNTL frauds into four types: segmented CNTL frauds, fully overlapped CNTL frauds, partially overlapped CNTL frauds, and combined CNTL frauds.

To combat NTL frauds, many schemes have been proposed including intrusion detection based methods [13]-[16], industrial control based methods [17], [18], physical methods [19]-[22], profiling based methods [23], [24], statistical methods [25], [26] and comparison based methods [3], [11], [27]-[30]. These schemes can detect NTL frauds and identify the tampered meters, but they cannot detect CNTL frauds since these methods do not consider the co-existing or collaborating fraudsters, and therefore cannot distinguish one from many fraudsters.

In this paper, a novel detector, called Colluded NonTechnical Loss Fraud Detection (CNFD), is proposed to address the CNTL fraud problem in Smart Grid. CNFD has two steps to detect CNTL frauds: 1) NTL fraud detection and 2) fraudster differentiation. In the NTL fraud detection 
step, CNFD quickly identifies the tampered meter within a group of meters. In the fraudster differentiation step, CNFD differentiates multiple fraudsters in the tampered meter. CNFD adapts Recursive Least Square (RLS) [31] to model fraudsters' behaviors using linear functions. Different fraudsters have different models to represent themselves. CNFD is lightweight and requires only a small amount of data. CNFD can even predict the behaviors of fraudsters which they may not realize by themselves. We have conducted various experiments to test the effectiveness and performance of CNFD. The experimental results show that CNFD can effectively detect four types of CNTL frauds and describe the behaviors of different fraudsters clearly.

The main contributions of this paper include:

- We recently discovered a potential type of frauds in Smart Gird, called the CNTL frauds;

- We study the features of different CNTL frauds and categorize them into four types;

- We propose a novel detector CNFD to detect CNTL frauds in Smart Grid;

- Our experiments show that CNFD is effective.

The rest of the paper is organized as follows. The problem definition, the CNTL attack model, and the four types of CNTL frauds are introduced in Section II. In Section III, we introduce the CNFD algorithm. In Section IV, experiments and experimental results are presented to show the effectiveness of CNFD. In Section V, we introduce related works and conclude the paper in Section VI.

\section{Colluded NTL Fraud}

In this section, we first define the problem that we are trying to solve, and then present the attack model of CNTL frauds. Furthermore, detailed analysis on the features of these frauds is then presented, and based on the analysis, we classify CNTL frauds into four types.

\section{A. Problem definition}

Alabama Power, a utility company headquartered in Birmingham, is responsible for providing electricity service for southern Alabama where Alabama is a state located in the southeast (SEC) region of USA. Consider a community with $N$ households in total served by Alabama Power. As shown in Fig. 1, these households have smart meters installed outside their houses, and each household has one smart meter. Some households may have multiple meters, but there is always one meter that is responsible for recording the total energy consumption and providing data for the billing purpose. Alabama Power keeps records of the total amount of electricity supplied to this community. However, the total billed amount is always smaller than the supplied amount if there are some compromised meters.

Alabama Power checks technical problems during the transmission and distribution processes. Electricity losses of power lines and transformers are deducted from the total loss. But the amount of loss is still large. Alabama Power suspects that at least one meter was tampered with in this community, and one or more than one customer are fraudsters who stole electricity. The problem is how to identify the tampered meters and fraudsters.

In this paper, we only consider the case that the total billed amount is smaller than the consumed amount, i.e., it is an NTL fraud; we do not consider the case that the total billed amount is larger than the consumed amount, i.e., it is not an NTL fraud.

\section{B. Attack model}

There are two types of fraudsters in general: inside fraudsters (fraudsters inside the households) and outside fraudsters (fraudsters outside the households). Inside fraudsters have the ability to physically tamper with smart meters, such as slowing down the meters using magnets. They also have the ability to impersonate smart meters using simulation software or overwrite the firmware of the meters. Outside fraudsters have the ability to remotely control smart meters, intercept the communication between smart meters and head-end systems in the utility, and extract encryption keys or passwords from network traffic. If the fraudsters are from the outside, the owner of the tampered meter may not realize the fraud. In a single tampered meter, the outside fraudsters and the inside fraudsters may co-exist.

As shown in Fig. 1, some smart meters are normal, while some other smart meters are tampered with. In a tampered meter, there may be one or multiple fraudsters. Collaborating fraudsters are those fraudsters who cooperate with each other to tamper with a meter and are aware of the existence of other fraudsters. Co-existing fraudsters are those fraudsters in a single tampered meter who just co-exist and are not aware of the existence of other fraudsters. Typically, collaborating fraudsters are either from the outside or from the inside. It is not common for the inside fraudsters to collaborate with the outside fraudsters. But for co-existing fraudsters, inside fraudsters may co-exist with outside fraudsters.

\section{Colluded NTL Fraud}

A CNTL fraud occurs when multiple fraudsters tamper with a meter so that the meter records less electricity than the consumed amount by the household, and the fraudsters gain illegal benefit by paying less money. After further analyzing these fraudsters, we find that they have different behaviors which they may not realize themselves. Moreover, the behaviors of how they collude the frauds can be different.

Sometimes, when a meter is tampered with by multiple fraudsters, these fraudsters may commit the malicious manipulation at different time segments. As shown in Fig. 2(a), we name this kind of CNTL frauds as segmented CNTL frauds. In a segmented CNTL fraud, fraudsters usually do not realize the existence of other fraudsters. Thus, these fraudsters are co-existing fraudsters, and are not collaborating fraudsters.

During most of the time, CNTL frauds are not segmented. Different fraudsters can manipulate the same meter at the same time, or at least part of the manipulation time overlaps. We call this kind of colluded NTL frauds as overlapped CNTL frauds. Overlapped CNTL frauds can be classified into partially overlapped CNTL frauds and fully overlapped 


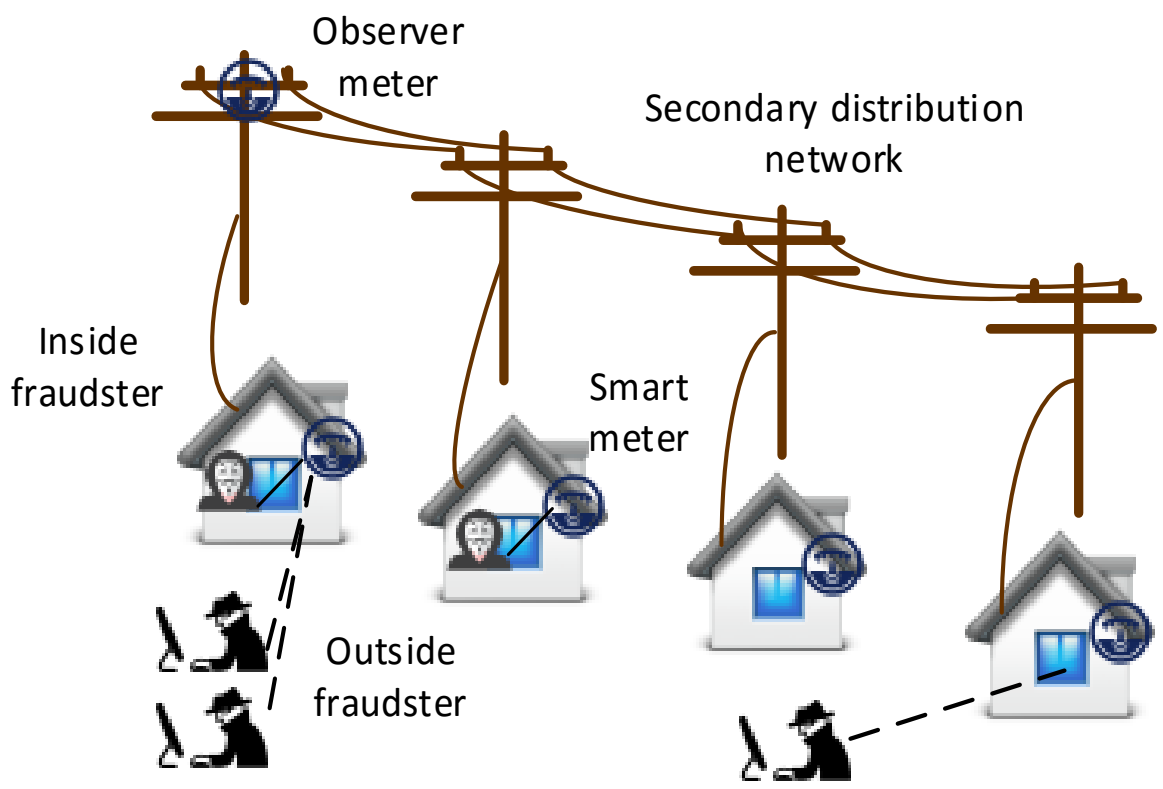

Fig. 1. The attack framework of CNTL frauds. Each household has a smart meter installed. The status of each meter could be 'normal' or 'tampered'. In a single tampered meter, there may be multiple fraudsters. These fraudsters could collaborate or just co-exist. The problem is that the total amount of billed electricity is much smaller than the total amount supplied.

CNTL frauds, as shown in Figs. 2(b) and (c), respectively. The difference between partially overlapped CNTL and fully overlapped CNTL is that there is one fraudster who overlaps all other fraudsters in a fully overlapped CNTL fraud. This feature can easily fool a detector and can make the detector believe that there is only one fraudster in this meter.

Some frauds are even more complicated since they have both segmented CNTL and overlapped CNTL, and we name them as combined CNTL frauds, shown in Fig. 2(d). In an overlapped CNTL fraud or a combined CNTL fraud, fraudsters may or may not realize the existence of other fraudsters. Thus, these fraudsters could be co-existing fraudsters, or they could also be collaborating fraudsters.

\section{CNTL FraUd DeteCtion}

In this section, we will introduce how to detect CNTL frauds using our proposed CNFD method, and the algorithm of CNFD is also presented.

\section{A. Observer meter}

We install an observer meter to record the total amount of electricity supplied to $n(n \leq N)$ households in this community. The observer meter is installed at the pole side keeping a distance to the properties of these households. Among these $n$ households, one or multiple meters are suspected to be tampered with. To guarantee the safety of the observer, we attach a tamper-resistant device to the observer and monitor it intensively using a camera. The tamper-resistant device is to protect the observer from cyber attacks. Camera surveillance is to protect the observer from any physical tampering. The observer meter is shown in Fig. 1. There are two reasons that we cannot secure all the meters using the way that we secure the observer. One reason is the limited budget. Another reason is privacy consideration [32]-[35].

The smart meters owned by the households are responsible for keeping records of the energy consumptions of the households. The metering system will automatically read these meters, including smart meters and the observer. The interval of meter reading is fixed, but the length of the interval is adjustable, e.g. from several minutes to several months. A typical interval is 15 minutes [1], [36]. Now, we have two set of known values. One set is the energy consumption reported by smart meters. Note that the reported value may be smaller than the amount of the actual energy consumption of the household due to the fraud. Another value is the total amount supplied to the $n(n \leq N)$ households. Our objective is to figure out which meters have CNTL frauds.

\section{B. Tampered meter detection}

CNFD includes two steps. The first step is to detect tampered meters. We list notations used in this paper as follows:

- $T_{j}: j$-th measurement time period;

- $m$ : the total number of measurements;

- $E_{i j}$ : the electricity actually consumed by Meter $i$, during the time period $T_{j}$;

- $E_{j}$ : the total electricity recorded at an observer meter, during the time period $T_{j}$;

- $E$ : the column vector of $E_{j}$ of all meters;

- $x_{i j}$ : the electricity recorded by Meter $i$, during the time period $T_{j}$;

- $X_{j}$ : the row vector of $x_{i j}$ of all meters, during the time period $T_{j}$;

- $X$ : the column vector of $X_{j}$;

- $a_{i j}$ : the coefficient of Meter $i$, during the time period $T_{j}$;

- $a_{i}$ : the coefficient vector of Meter $i$; 


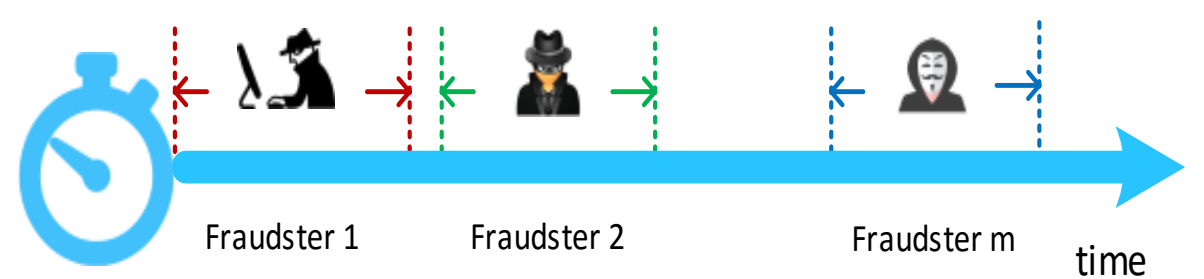

(a) Segmented CNTL fraud

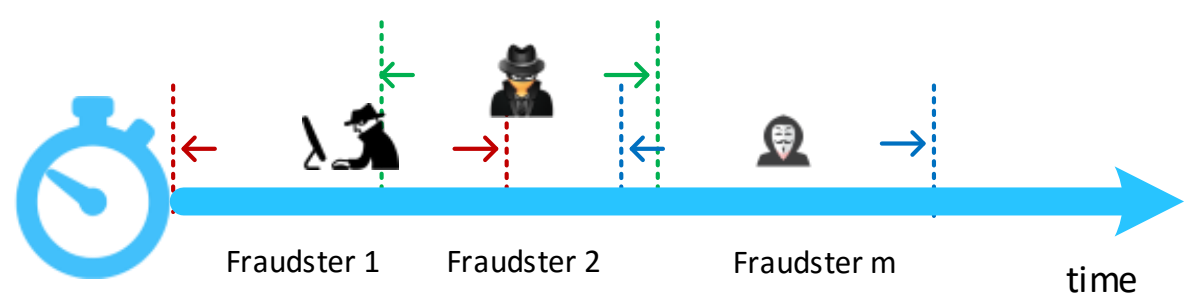

(b) Partially overlapped CNTL fraud

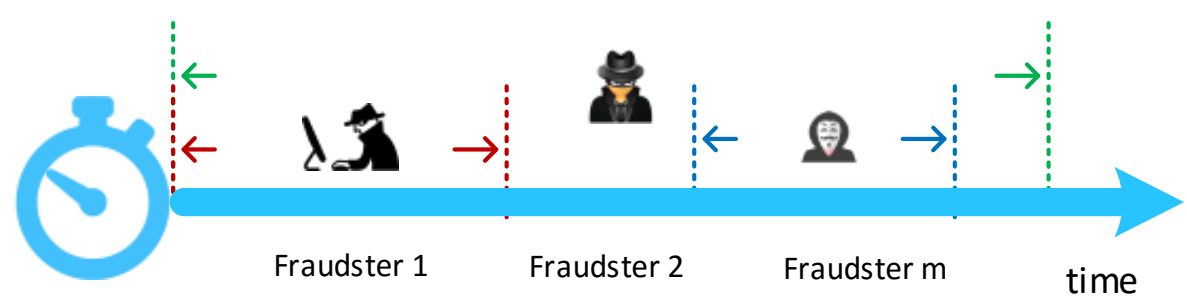

(c) Fully overlapped CNTL fraud

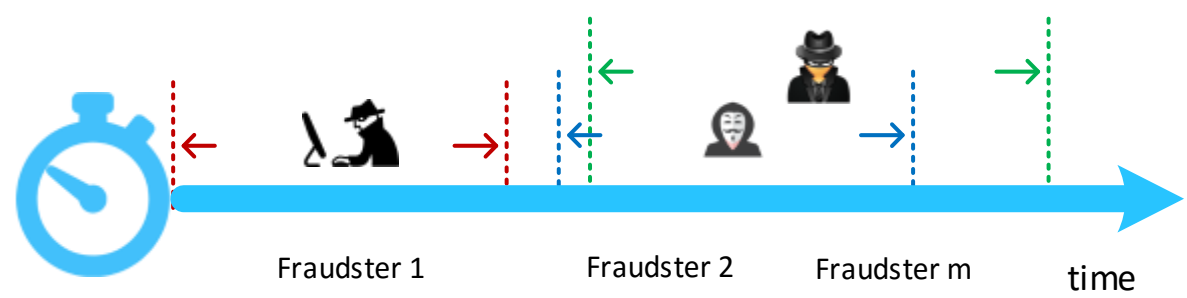

(d) Combined CNTL fraud

Fig. 2. Four different types of CNTL frauds that we discovered. a) Segmented CNTL fraud; b) Partially overlapped CNTL fraud; c) Fully overlapped CNTL fraud; d) Combined CNTL fraud.

- $A$ : the row vector of $a_{i}$ of all meters;

- $S_{i}$ : the coefficient set of all different fraudsters in Meter $i$; - $e$ : the user defined error.

The values of $\left(E_{i j}, j=1,2, \cdots, m\right)$ are not available although the values of $\left(x_{i j}, j=1,2, \cdots, m\right)$ are available. For a value pair $\left(x_{i j}, E_{i j}\right)$, there is a corresponding point, such as Point B, at the coordinate as shown in Fig. 3. Each meter has a group of points of its own, and we can find a line closet to these points to represent this meter. Let us denote this line as:

$$
f_{i}(x)=a_{i} x .
$$

Note that each line starts from $(0,0)$, since the recorded value must be 0 if the consumed energy is 0 .

If Meter $i$ is a normal meter, either $E_{i j} / x_{i j}=1$ holds or $\left|E_{i j} / x_{i j}-1\right|$ is very small. If $\left|E_{i j} / x_{i j}-1\right|$ is large, this indicates that Meter $i$ is an abnormal (tampered) meter. Define $\alpha_{i j}=E_{i j} / x_{i j}$ which indicates the accuracy ratio, i.e., the error committed by Meter $i$ during the period $j$. For simplicity and without losing generality, we assume that if Meter $i$ is a normal meter, its $\alpha_{i j}$ should be in the range as follows:

$$
\alpha_{i j} \in\left[\alpha_{\min }, \alpha_{\max }\right],(1 \leq i \leq n) .
$$

The accuracy of metering is affected by many factors, including connection type, power factor, current, standards in different countries, etc. A typical metering system accuracy is $\pm 1.55 \%$ [37]. To accommodate rounding error and data noise, we choose $\pm 2 \%$ as the accuracy class, i.e., $\alpha_{\min }=0.98$ and $\alpha_{\max }=1.02$. We also assume that if one meter is tampered with (abnormal), we have $\alpha_{i j}>\alpha_{\max }$. In other words, we assume that all of the normal meters have the same accuracy range as the above, and all of the abnormal (tampered) meters have higher accuracy values above the range. Generally speaking, $\alpha_{i j}$ could be less than $\alpha_{m i n}$, and 


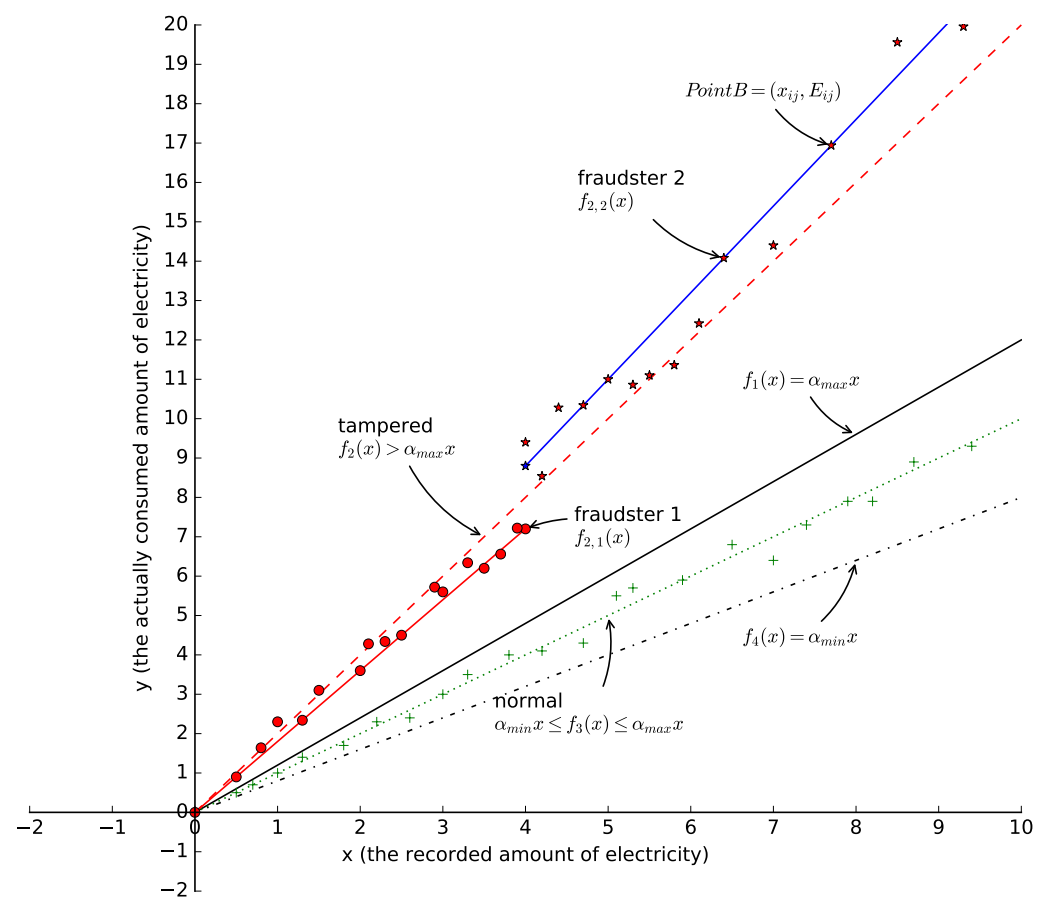

Fig. 3. Modeling the behavior of fraudsters mathematically and differentiating them. Each value pair, e.g. (the recorded amount of electricity, the actually consumed amount of electricity) has a point to represent it at the coordinate. Each meter has a dataset containing a series of value pairs obtained from several measurements. The dataset has a set of corresponding points. In CNFD, a line which represents the set of points is used to represent the meter. For tampered meters, their lines are above the line of $f_{1}(x)=\alpha_{\max } x$. For normal meters, their lines are between the lines of $f_{1}(x)=\alpha_{m a x} x$ and $f_{4}(x)=\alpha_{m i n} x$. Some lines can be reshaped into multiple lines.

this means that the billing amount of electricity is larger than the consumed amount so that the customer has to pay more money. The customer herself (himself) will never tamper with a meter in this way. It only happens when a meter is manipulated by outside attackers and the meter readings are altered to be higher than the actual values. However, it is not an NTL fraud in this situation, and it is out of the scope of this paper.

As shown in Fig. 3, a tampered meter has a line above the line of $f_{1}(x)=\alpha_{\max } x$, and a normal meter has a line between the lines of $f_{1}(x)=\alpha_{\max } x$ and $f_{4}(x)=\alpha_{\min } x$. A line below the line of $f_{4}(x)$ is a sign of working error. We can figure out which meters are tampered with and obtain the coefficient vector $A$. We notice that

$$
E_{j}=\sum_{i=1}^{n} E_{i j}
$$

and $E_{j}$ is available. We re-write it as follows:

$$
E_{j}=\sum_{i=1}^{n} a_{i} x_{i j} .
$$

Let $X_{j}$ be the values of the $j$-th measurement. Let $A$ be the array of all coefficients. We have:

$$
A=\left(a_{1}, a_{2}, \ldots, a_{n}\right) .
$$

$$
X_{j}=\left(x_{1 j}, x_{2 j}, \ldots, x_{n j}\right) .
$$

Suppose that we have an estimation of $A$ after $j-1$ times of measurements, $A_{j-1}$. Now we obtain a new measurement $X_{j}$ and $E_{j}$, and want to update the estimation with this new measurement. According to the theory of Recursive Least Square (RLS), to get a new estimation $A_{j}$, we have:

$$
A_{j}=A_{j-1}+W_{j}\left(E_{j}-X_{j} A_{j-1}\right),
$$

where

$$
\begin{gathered}
W_{j}=P_{j-1} X_{j}^{T}\left(\lambda+X_{j} P_{j-1} X_{j}^{T}\right)^{-1}, \\
P_{j}=\left(I-W_{j} X_{j}\right) P_{j-1} / \lambda, \\
P_{j}^{-1}=\lambda P_{j-1}^{-1}+X_{j}^{T} X_{j} .
\end{gathered}
$$

Here, $W$ is a weight array. $I$ is the identity matrix. $P$ is the degree of precision of the measurement, and is initialized with $P_{0}=k I$, where $k$ is a high value constant. $\lambda$ is a forgetting factor with a value between 0 and $1(0<\lambda \leq 1)$. In order to reduce the influence of old values, we should choose a lower value of $\lambda$.

Let us set $j$ as 1 , since we have no historical data, and thus have no knowledge of $A$. As shown in Alg. 1, the process is repeated until the value of $A$ does not change. Then, each $a_{i}$ in $A$ is compared to $\alpha_{\min }$ and $\alpha_{\max }$. If $a_{i}$ is larger than 
$\alpha_{\max }$, Meter $i$ is identified as 'tampered'. Then the value of $i$ is assigned to $t$.

\section{Fraudster differentiation}

The second step of CNFD is fraudster differentiation. We identified the tampered meter, Meter $t$, in the first step, and the value of the coefficient vector $A$ is known. In this step, CNFD will try to find out whether there are multiple fraudsters in the tampered meter and differentiate them. An illustrative example is shown in Fig. 3. In the first step, the red points are used to generate a red line, $f_{2}(x)$, which is above the line of $f_{1}(x)=\alpha_{\max } x$. Thus, the meter represented by the red points is identified as the tampered meter. After further analysis, we find that the red points can be reshaped into two lines which are $f_{2,1}(x)$ and $f_{2,2}(x)$. The red dot points are closer to $f_{2,1}(x)$ rather than $f_{2}(x)$, and the red star points are closer to $f_{2,2}(x)$ rather than $f_{2}(x)$. In fact, there are two fraudsters and their behaviors are represented by $f_{2,1}(x)$ and $f_{2,2}(x)$, respectively.

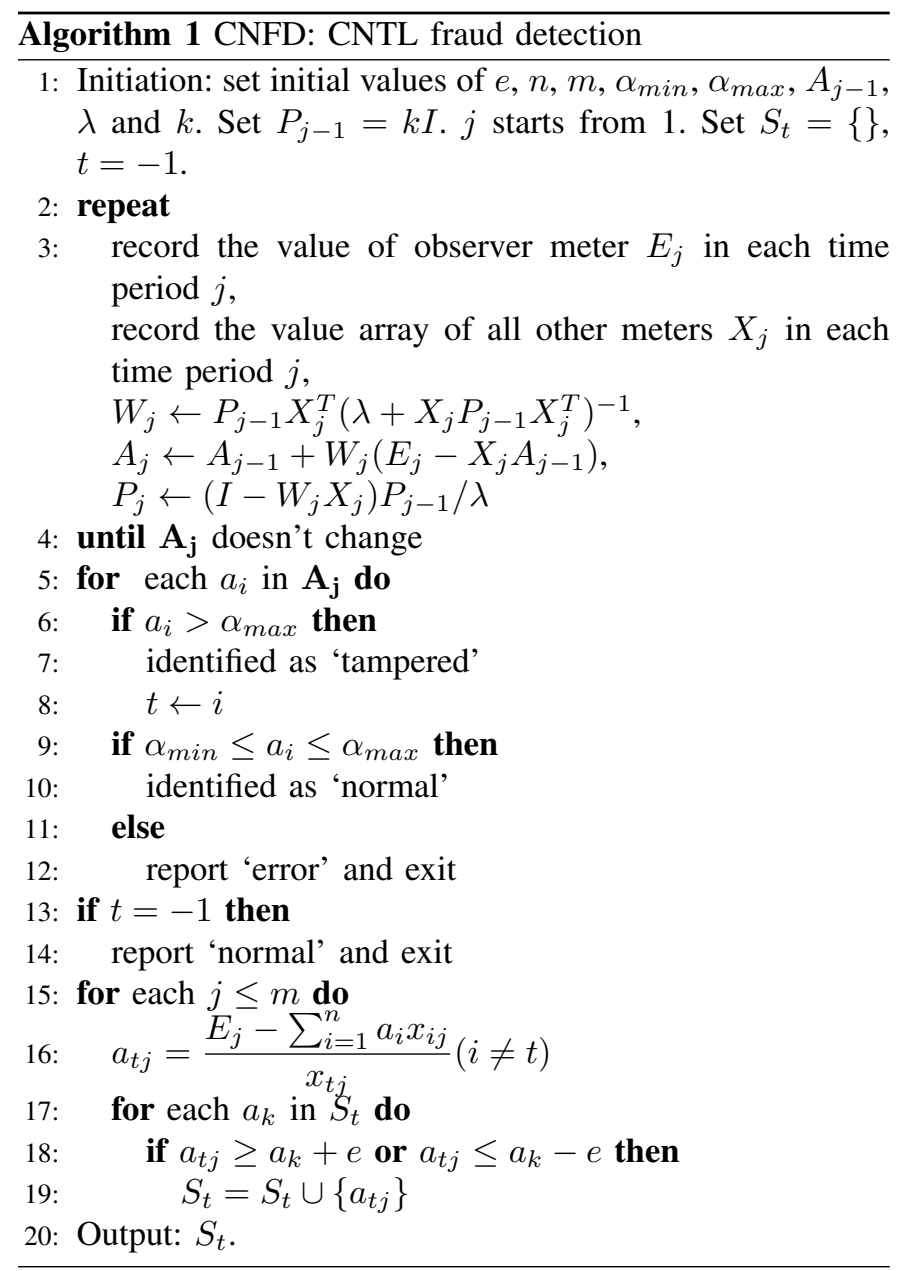

The algorithm of CNFD is shown in Alg. 1, which includes the two steps. The codes from Line 15 to Line 20 in Alg. 1 show the process of fraudster differentiation. The basic idea of fraudster differentiation is to calculate the coefficients at every interval after isolating the tampered meter, and analyze the similarity of these values within a given error $e$. Finally, these values are identified as belonging to different fraudsters. Here, $e$ is a given value of error that defined by users. $S_{t}$ contains the output coefficients of all the fraudsters in Meter $t$. $S_{t}$ is set initially to be an empty set. If the output coefficients in $S_{t}$ are very similar, within the range of $e$, it means that there is only one fraudster. In other words, it is not a CNTL fraud.

\section{EXPERIMENTS}

In this section, we will introduce four experiments to show the effectiveness of CNFD. These four experiments show results of detecting segmented CNTL frauds, fully overlapped CNTL frauds, partially overlapped CNTL frauds, and combined CNTL frauds, respectively. The user defined error $e$ is set to $0.5 \%$. There are 30 measurement records in each experiment, but we only list the first 20 records to save space. The value of $k$ is $100, \lambda$ is 0.01 , and $a_{i}$ is 2 . We set the measurement accuracy class as $2 \%$, i.e., $\alpha_{\min }=0.98$ and $\alpha_{\max }=1.02$. If a meter's line falls in the range between $y=0.98 x$ and $y=1.02 x$, it is a normal meter. Any line of a meter that is above $y=1.02 x$ is a tampered meter's line. It indicates that an error occurs if there is any line of a meter is below $y=0.98 x$. The data used in the experiments are synthetic data. We obtained normal electricity consumption data based on a real-world dataset [38], and increased the consumption of some meters randomly. The measurement interval is one month.

\section{A. Experiment 1: segmented CNTL}

In the first experiment, there are 10 meters under the observation of one observer meter. The recorded values and the total supplied value of electricity are listed in Table I.

As shown in Fig. 4, this is the tampered meter detection process in Exp. 1, which shows that Meter 4 is a tampered meter and the line of $y=2.216 x$ represents its fraudster's behavior. After further analysis, we find that there are two fraudsters in this meter. Their behaviors are represented by $y=1.22 x$ and $y=2.22 x$, which are shown in Fig. 5. Each of the two fraudsters dominates a time segment of the whole process, and therefore it is a segmented CNTL fraud.

\section{B. Experiment 2: fully overlapped CNTL}

In the second experiment, there is one observer meter, and it monitors 10 other meters. We list the first 20 measurement records in Table II.

As shown in Fig. 6, this is the detection process in Exp. 2. The coefficient of Meter 2 converges to 2.51 while the coefficients of other meters converge to 1 , and this indicates that Meter 2 is a tampered meter. We further analyze this meter using CNFD, and we find that this fraud is colluded by two fraudsters. However, the behavior of Fraudster 1 is fully covered by Fraudster 2. Therefore, it is a fully overlapped CNTL fraud. As shown in Fig. 7, the behaviors of these two fraudsters are represented by $y=1.33 x$ and $y=2.51 x$, respectively. 
TABLE I

THE RECORDED ELECTRICITY CONSUMPTION (KWH) IN EXPERIMENT 1

\begin{tabular}{|c|c|c|c|c|c|c|c|c|c|c|}
\hline Meter 1 & 2 & 3 & 4 & 5 & 6 & 7 & 8 & 9 & 10 & Observer \\
\hline 2790 & 3970 & 5540 & 2960 & 3440 & 9950 & 5550 & 4340 & 5740 & 6700 & 51631.2 \\
\hline 3400 & 4200 & 6510 & 3620 & 2360 & 9970 & 6030 & 3590 & 5310 & 6840 & 52626.4 \\
\hline 2910 & 4460 & 7650 & 3910 & 3240 & 1017 & 6290 & 2500 & 5710 & 6980 & 54680.2 \\
\hline 3370 & 3760 & 6880 & 3180 & 4060 & 1089 & 6190 & 6230 & 7670 & 8080 & 61009.6 \\
\hline 3690 & 3940 & 6600 & 3580 & 6090 & 9780 & 6830 & 3280 & 6200 & 5320 & 56097.6 \\
\hline 2350 & 5170 & 7150 & 3540 & 6600 & 10530 & 7330 & 4690 & 6270 & 6920 & 64868.8 \\
\hline 3820 & 3390 & 7310 & 4290 & 3130 & 9170 & 6430 & 4270 & 6270 & 6560 & 59873.8 \\
\hline 3120 & 4930 & 5550 & 2180 & 4110 & 9790 & 5820 & 5200 & 3350 & 7570 & 54279.6 \\
\hline 4580 & 4320 & 5720 & 3040 & 4480 & 9440 & 6350 & 4250 & 6320 & 7230 & 59438.8 \\
\hline 2300 & 4220 & 4560 & 3920 & 3280 & 9830 & 5500 & 4860 & 6630 & 5840 & 55722.4 \\
\hline 3350 & 3900 & 5850 & 2990 & 6120 & 8760 & 5650 & 4720 & 6670 & 6220 & 57877.8 \\
\hline 3320 & 4180 & 5550 & 2990 & 6620 & 8470 & 6800 & 3580 & 5900 & 5290 & 56347.8 \\
\hline 2780 & 3310 & 5800 & 3870 & 4850 & 8560 & 6700 & 3210 & 3900 & 4030 & 51731.4 \\
\hline 2980 & 4900 & 6560 & 2520 & 6130 & 10350 & 8000 & 3620 & 4660 & 4900 & 57694.4 \\
\hline 3010 & 4100 & 7210 & 4460 & 5480 & 9530 & 8260 & 4080 & 5620 & 7260 & 64451.2 \\
\hline 4260 & 3470 & 7290 & 5330 & 4630 & 9790 & 8460 & 3650 & 4730 & 6920 & 65032.6 \\
\hline 3610 & 3640 & 5740 & 4140 & 4870 & 9730 & 6630 & 3590 & 5870 & 7420 & 60290.8 \\
\hline 3190 & 4690 & 5930 & 4140 & 6240 & 10710 & 6870 & 4620 & 6060 & 6800 & 64300.8 \\
\hline 3060 & 5800 & 6450 & 3790 & 3290 & 11190 & 7510 & 4700 & 5080 & 6760 & 62253.8 \\
\hline 3660 & 4050 & 7010 & 3610 & 5160 & 9810 & 6670 & 5520 & 6250 & 6440 & 62584.2 \\
\hline
\end{tabular}

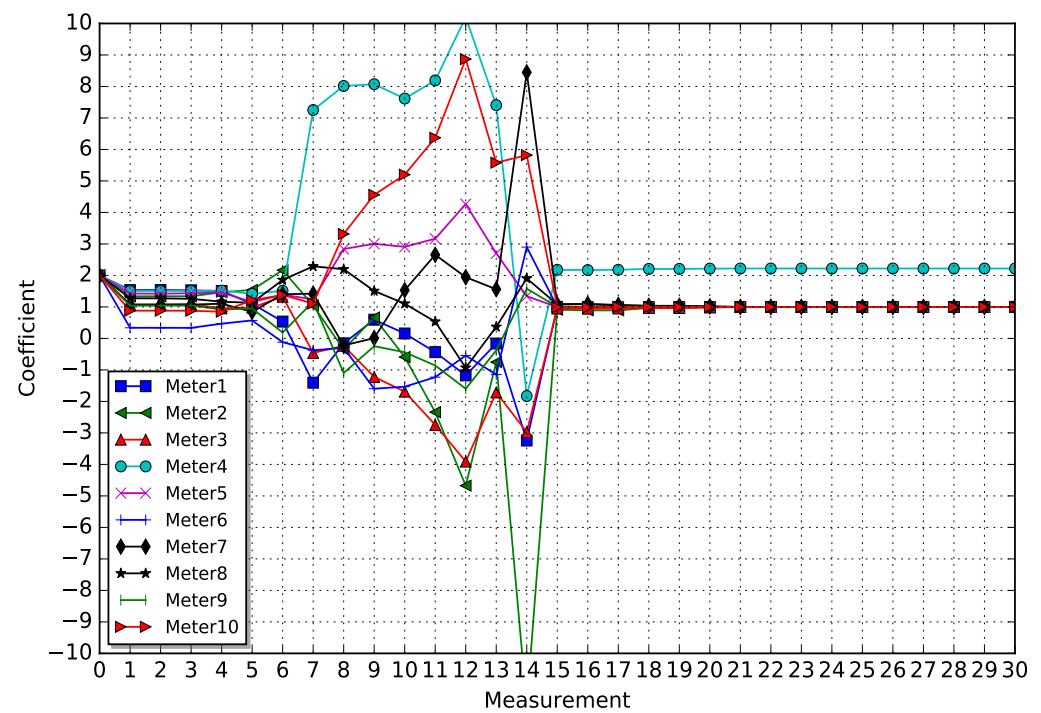

Fig. 4. The tampered meter detection process in Exp. 1: the converging process of the coefficients of 10 meters. Among these 10 meters, the coefficient of Meter 4 converges to 2.216 while others converge to 1 , and this indicates that Meter 4 is a tampered meter.

\section{Experiment 3: partially overlapped CNTL}

In this experiment, there are still 10 meters which are monitored by one observer meter. Table III shows 20 measurements of data of the recorded values of electricity.

The tampered meter detection result is shown in Fig. 8. Coefficients of all other meters converge to 1 while the coefficient of Meter 3 converges to 3.11, and this indicates that Meter 3 is a tampered meter. We analyze Meter 3 using CNFD, and find that there are two fraudsters who manipulated this meter. Their manipulation behaviors overlapped in some time segments, but not in the whole process. Thus, it is a partially overlapped CNTL fraud. These two fraudsters' behaviors are $y=1.88 x$ and $y=3.11 x$ in terms of mathematics, which are shown in Fig. 9.

\section{Experiment 4: combined CNTL}

This is the last experiment. In this experiment, we still employ 10 meters and an observer. Table IV shows their recorded data in 20 measurements.

The result of the first step, tampered meter detection, of CNFD is shown in Fig. 10. Meter 6 is a tampered meter because the curve of its coefficients finally converges to 1.972 . The other 9 meters are normal since the curves of their coefficients finally converge to 1 . In the second step, three 


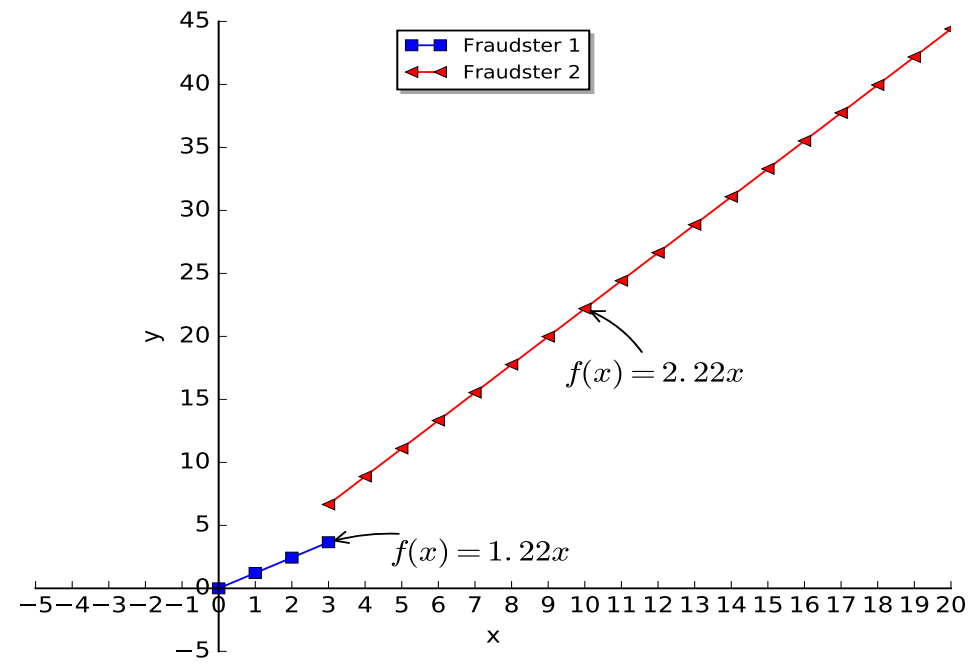

Fig. 5. The final detection result of CNFD in Exp. 1. CNFD detection shows that there are two different fraudsters who collude the fraud in Meter 4.

TABLE II

THE RECORDED ELECTRICITY CONSUMPTION (KWH) IN EXPERIMENT 2

\begin{tabular}{|c|c|c|c|c|c|c|c|c|c|c|}
\hline Meter 1 & 2 & 3 & 4 & 5 & 6 & 7 & 8 & 9 & 10 & Observer \\
\hline 3500 & 3480 & 6300 & 2990 & 3640 & 9200 & 5240 & 4270 & 5150 & 6500 & 51418.4 \\
\hline 3420 & 3960 & 6730 & 3250 & 2950 & 9580 & 5770 & 3380 & 5790 & 6880 & 57689.6 \\
\hline 2830 & 4380 & 7210 & 3800 & 3060 & 9480 & 5740 & 2850 & 5740 & 6490 & 53025.4 \\
\hline 3040 & 4060 & 7640 & 3020 & 3800 & 1109 & 6930 & 6610 & 7760 & 8020 & 63309.8 \\
\hline 4170 & 3510 & 6370 & 3740 & 6000 & 9970 & 7380 & 3380 & 6240 & 5350 & 61410.1 \\
\hline 2640 & 4800 & 7530 & 4030 & 6620 & 10120 & 7760 & 4090 & 5890 & 6240 & 66968 \\
\hline 3210 & 3830 & 7860 & 3750 & 2610 & 9600 & 6570 & 4400 & 5720 & 6490 & 55303.9 \\
\hline 2860 & 4240 & 5850 & 2460 & 4040 & 9840 & 6100 & 4270 & 3470 & 7390 & 56922.4 \\
\hline 3760 & 4200 & 5580 & 3610 & 3880 & 9930 & 6040 & 4350 & 7080 & 7100 & 56916 \\
\hline 2750 & 4230 & 5030 & 3720 & 3550 & 10540 & 5270 & 4970 & 6730 & 6020 & 54205.9 \\
\hline 3440 & 3830 & 5530 & 3070 & 6790 & 9180 & 5860 & 3930 & 6090 & 5900 & 59403.3 \\
\hline 3520 & 3680 & 6190 & 3120 & 6360 & 7820 & 6730 & 3540 & 6120 & 4700 & 57336.8 \\
\hline 2120 & 3150 & 6300 & 4160 & 4480 & 7940 & 7320 & 3190 & 3690 & 3930 & 51036.5 \\
\hline 2940 & 4930 & 6610 & 2110 & 6370 & 10520 & 7890 & 4080 & 4640 & 5330 & 62864.3 \\
\hline 3400 & 3910 & 6940 & 4500 & 5890 & 9530 & 7880 & 3980 & 4640 & 6900 & 63474.1 \\
\hline 4410 & 3320 & 7130 & 5050 & 4860 & 10120 & 7950 & 3300 & 5530 & 7500 & 64183.2 \\
\hline 3250 & 3800 & 6060 & 4990 & 4680 & 9300 & 6780 & 3370 & 6130 & 7080 & 61178 \\
\hline 3120 & 4780 & 6010 & 4290 & 5780 & 10240 & 7670 & 4060 & 6470 & 7010 & 66647.8 \\
\hline 2370 & 5810 & 7070 & 3450 & 3230 & 11480 & 7050 & 4220 & 5380 & 6900 & 65733.1 \\
\hline 3580 & 3580 & 6710 & 3670 & 5010 & 10270 & 7170 & 5030 & 6420 & 7130 & 63975.8 \\
\hline
\end{tabular}

different fraudsters are identified, and they are represented by $y=1.45 x, y=1.19 x$, and $y=1.98 x$, respectively. Fraudster 3 partially overlaps Fraudster 2, while Fraudster 1 and Fraudster 2 are segmented. Thus, it is a combined CNTL fraud shown in Fig. 11.

\section{RELATED WORK}

The physical method has the longest history in combating NTL fraud. In the traditional power grid, surveillance staffs are hired to examine power lines and get rid of bypasses routinely [39]. In Smart Grid, monitoring is still a good choice to deal with bypassing and other types of physical tampering. In the paper [19], video surveillance by drones is used to analyze the suspected segments in the feeder line, and a control center is employed to identify NTL frauds and notify the nearest vigilance staff. Some research focuses on designing secure modules installed along each smart meter. The paper [22] introduces a smart prepaid metering system employing a special energy meter which can protect against meter bypassing. The main drawback of these physical methods is that they either cannot pinpoint a tampered meter, or they are expensive when they can pinpoint a tampered meter. In Smart Grid, automatic power line examination cannot only help to maintain the functionality of the power line but also help to address NTL fraud problems. A power line impedance technique is proposed in the papers [20], [21] to locate tampered meters. By 


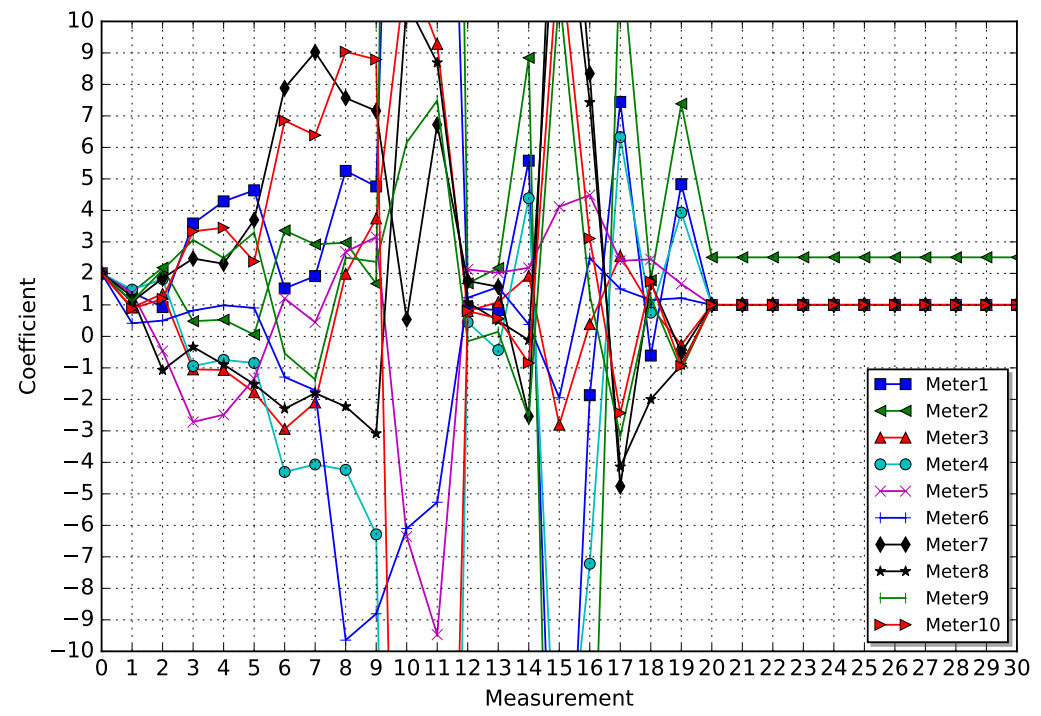

Fig. 6. The tampered meter detection process in Exp. 2: the converging process of the coefficients of 10 meters. Among these 10 meters, the coefficient of Meter 2 converges to 2.51 while others converge to 1 , and this indicates that Meter 2 is a tampered meter.

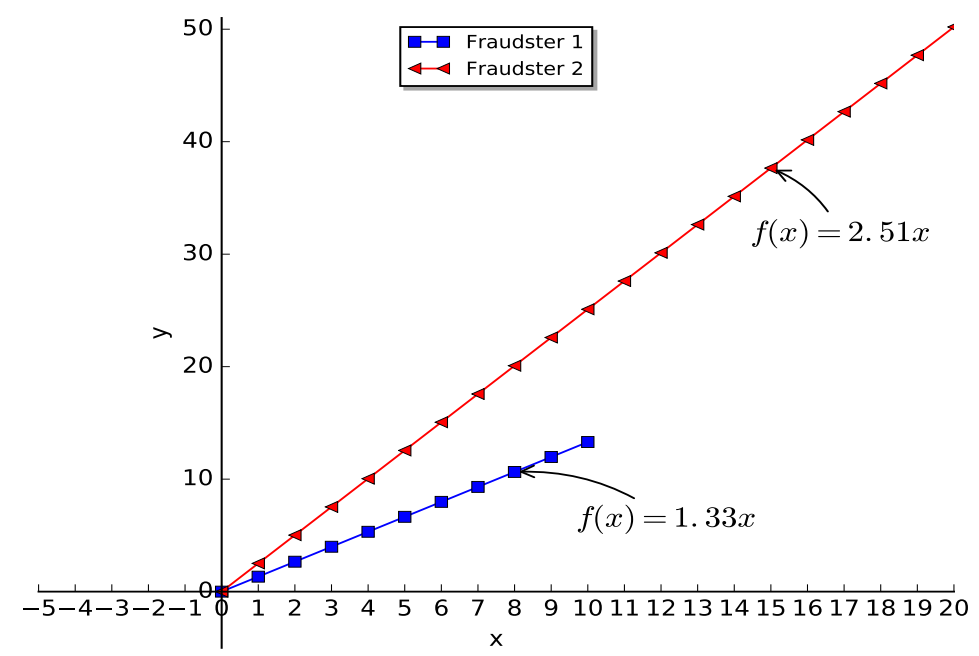

Fig. 7. The final detection result of CNFD in Exp. 2. CNFD detection shows that two different fraudsters collude the fraud on Meter 2.

using two different operating frequencies, the phase angle of the transmission lines and the impedance values are measured and used to locate the tampered meters.

Physical methods have three shortcomings. The first shortcoming is that the monitoring methods are expensive and inefficient. The monitoring methods need humans involved. Video surveillance still needs staff to watch these videos. The second shortcoming is that they are mainly used to detect NTL frauds committed via physical methods, such as power line bypassing. They cannot detect NTL frauds committed via cyber attacks, such as manipulating a billing message and changing the values of the billing amount. The third shortcoming is that customers easily complain about privacy invasion [33], [40]-[42].

Profiling is a typical method used to detect NTL frauds, and a method using profiling typically obtains energy consumption profiles of different types of users. The mostly used technologies are Artificial Intelligence (AI) enabled technologies, such as Pattern Recognition [43], [44], Machine Learning [45], [46], Data Mining [47], etc. In the paper [23], Support Vector Machines (SVMs) are trained with energy consumption data that represent all possible types of energy theft. A dataset that contains the energy consumption patterns of users is generated based on historical data. Then energy consumption profiles of these customers are built and classified according to some given rules, and the suspicious profiles are grouped. The 
TABLE III

THE RECORDED ELECTRICITY CONSUMPTION (KWH) IN EXPERIMENT 3

\begin{tabular}{|c|c|c|c|c|c|c|c|c|c|c|}
\hline Meter 1 & 2 & 3 & 4 & 5 & 6 & 7 & 8 & 9 & 10 & Observer \\
\hline 3440 & 4220 & 6220 & 2930 & 3690 & 9130 & 5270 & 4400 & 5140 & 6140 & 56053.6 \\
\hline 3550 & 4180 & 7050 & 4190 & 2690 & 9670 & 6000 & 3300 & 5840 & 6810 & 59484 \\
\hline 3530 & 4650 & 7600 & 4140 & 3400 & 10140 & 5710 & 2760 & 6330 & 6440 & 61388 \\
\hline 3330 & 3470 & 7560 & 2720 & 4030 & 10360 & 6600 & 5900 & 7560 & 7630 & 75111.6 \\
\hline 4170 & 3650 & 6700 & 3780 & 5410 & 9290 & 6910 & 3630 & 5710 & 5900 & 69287 \\
\hline 2090 & 5410 & 7930 & 3570 & 6480 & 10260 & 7140 & 3960 & 5750 & 6260 & 75582.3 \\
\hline 3870 & 3550 & 7950 & 4230 & 3080 & 9400 & 6290 & 4750 & 5980 & 6180 & 72054.5 \\
\hline 3440 & 4390 & 6370 & 2220 & 4430 & 9210 & 6340 & 5160 & 3230 & 7520 & 57915.6 \\
\hline 4530 & 4150 & 5550 & 3340 & 4730 & 9700 & 5780 & 3790 & 7240 & 6920 & 60614 \\
\hline 2230 & 3900 & 4560 & 4020 & 3080 & 10510 & 5890 & 5220 & 6280 & 5230 & 54932.8 \\
\hline 2970 & 3820 & 6010 & 3780 & 5920 & 9030 & 6090 & 4480 & 6470 & 5720 & 66971.1 \\
\hline 2980 & 3770 & 6360 & 2730 & 6220 & 8460 & 6570 & 3740 & 5910 & 4400 & 64559.6 \\
\hline 2440 & 3380 & 5760 & 3830 & 4670 & 8010 & 6390 & 3570 & 3240 & 3680 & 57123.6 \\
\hline 3170 & 4750 & 6630 & 2670 & 5740 & 10090 & 8010 & 3630 & 5260 & 4660 & 68599.3 \\
\hline 3080 & 3370 & 7190 & 3930 & 5540 & 10260 & 8220 & 3790 & 5440 & 6480 & 72470.9 \\
\hline 4470 & 3950 & 6560 & 5080 & 5050 & 9280 & 8640 & 3100 & 4990 & 6890 & 71851.6 \\
\hline 3560 & 4460 & 5770 & 4880 & 4670 & 9460 & 6210 & 2940 & 5560 & 7010 & 66694.7 \\
\hline 3590 & 5040 & 6500 & 4430 & 6200 & 10390 & 7680 & 4310 & 5920 & 7000 & 74775 \\
\hline 2900 & 5440 & 6970 & 3080 & 3940 & 10850 & 7200 & 4750 & 5520 & 7200 & 72556.7 \\
\hline 3880 & 4360 & 7380 & 2730 & 4880 & 9840 & 7090 & 4980 & 6260 & 7230 & 74201.8 \\
\hline
\end{tabular}

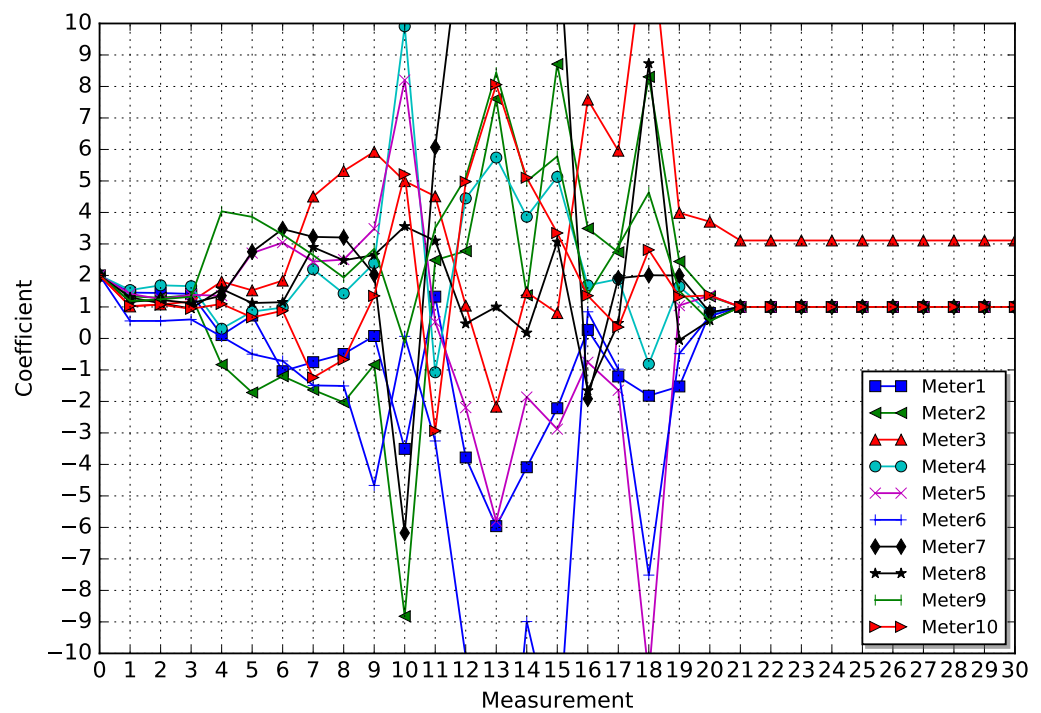

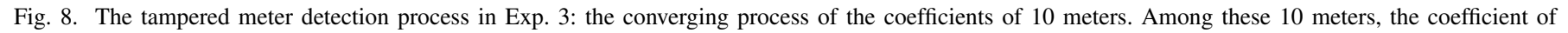
Meter 3 converges to 3.11 while others converge to 1 , which indicates that Meter 3 is a tampered meter.

authors in [24] introduce how to deal with the characterization of customers in utility companies to detect NTL frauds. They propose a new framework to find relevant knowledge of the particular characteristics of the customers.

Profiling methods have three drawbacks. The first drawback is that these methods often need lots of data to generate user profiles. The second drawback is that they are sensitive to variations, such as traveling or buying a new home appliance. The third drawback is that they can only detect frauds that have known patterns.

Comparison based methods [11], [27]-[30], [48] are to record a correct value of energy usage and compare the reading of a meter with this value. We have proposed NFD [29] and
FNFD [30], [48] which are comparison based methods. Both NFD and FNFD use an observer meter to record a correct value of energy usage for the purpose of comparison. The benefit of comparison based methods is that they usually require a small amount of data and lightweight. Both NFD and FNFD can detect NTL frauds, but they cannot detect CNTL fraud. NFD employs polynomial functions to build models, and thus it is more accurate than FNFD. FNFD employs linear functions to build models, and thus it is faster than NFD. Note that a short version of this paper was presented in the conference [12]. 


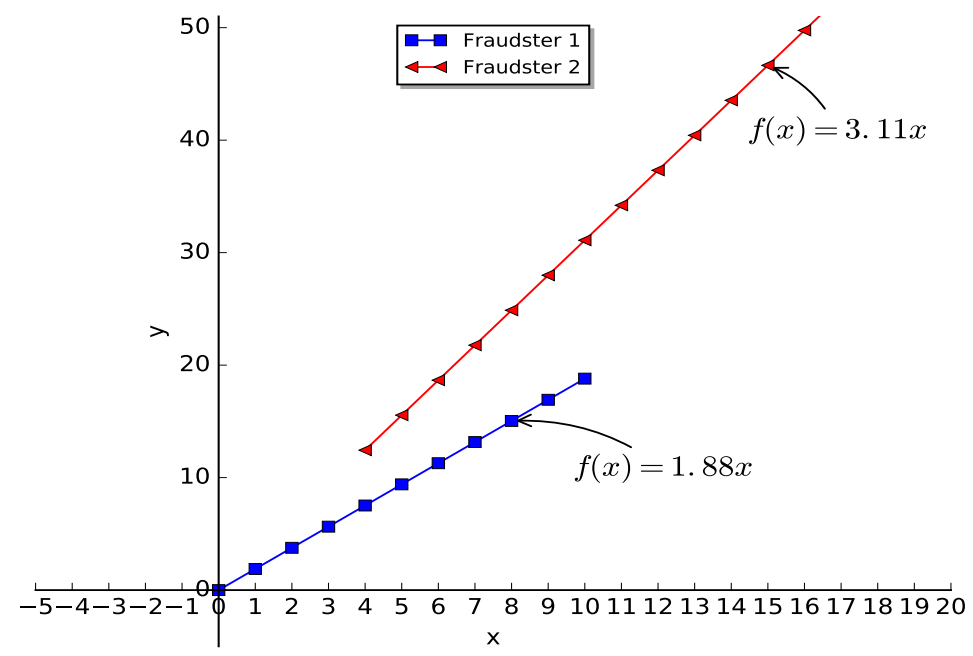

Fig. 9. The final detection result of CNFD in Exp. 3. CNFD detection shows that there are two different fraudsters who collude the fraud in Meter 3.

TABLE IV

THE RECORDED ELECTRICITY CONSUMPTION (KWH) IN EXPERIMENT 4

\begin{tabular}{|c|c|c|c|c|c|c|c|c|c|c|}
\hline Meter 1 & 2 & 3 & 4 & 5 & 6 & 7 & 8 & 9 & 10 & Observer \\
\hline 2920 & 3590 & 6470 & 2300 & 3490 & 9550 & 5530 & 4110 & 5830 & 6310 & 54397.5 \\
\hline 3560 & 3710 & 6980 & 3930 & 2650 & 9070 & 6280 & 2920 & 5440 & 6320 & 54941.5 \\
\hline 3100 & 4540 & 7140 & 3160 & 3230 & 9600 & 6380 & 2210 & 6020 & 6540 & 56240 \\
\hline 2660 & 4350 & 6860 & 3230 & 3540 & 10410 & 6800 & 6200 & 8210 & 7430 & 64374.5 \\
\hline 3380 & 4430 & 6100 & 3060 & 5220 & 9330 & 7380 & 3800 & 6510 & 5510 & 56492.7 \\
\hline 2240 & 5260 & 7360 & 4110 & 6740 & 10370 & 7270 & 4740 & 5820 & 6770 & 70842.6 \\
\hline 3670 & 3500 & 7920 & 4300 & 2570 & 9590 & 6260 & 4040 & 6330 & 6850 & 64428.2 \\
\hline 3000 & 4530 & 6250 & 2940 & 3760 & 9740 & 6500 & 4530 & 3280 & 7590 & 53970.6 \\
\hline 4590 & 4600 & 5600 & 3560 & 4270 & 9230 & 5980 & 4130 & 6650 & 7140 & 57503.7 \\
\hline 2920 & 4210 & 5150 & 3430 & 3190 & 10260 & 5460 & 4680 & 6910 & 5710 & 53869.4 \\
\hline 3640 & 3260 & 6150 & 3270 & 6430 & 9200 & 5860 & 4740 & 6540 & 5630 & 63736 \\
\hline 3050 & 3680 & 5430 & 3440 & 6470 & 8020 & 7190 & 3560 & 6610 & 4610 & 59919.6 \\
\hline 2220 & 3140 & 5930 & 4180 & 4940 & 8550 & 6760 & 2890 & 3710 & 3700 & 54399 \\
\hline 2720 & 4710 & 5990 & 2380 & 6340 & 10090 & 7620 & 3900 & 5160 & 4680 & 63478.2 \\
\hline 3480 & 3870 & 7280 & 4670 & 5270 & 9500 & 8380 & 3980 & 5220 & 6920 & 67880 \\
\hline 4228 & 3948 & 6678 & 5348 & 4780 & 10188 & 8088 & 3668 & 4818 & 7480 & 68976.4 \\
\hline 3840 & 3750 & 6090 & 4710 & 4870 & 9570 & 6840 & 3110 & 6270 & 7350 & 65778.6 \\
\hline 3740 & 5100 & 6410 & 4850 & 5570 & 9770 & 7430 & 4310 & 5670 & 6920 & 69344.6 \\
\hline 3060 & 5520 & 7180 & 3630 & 3900 & 10590 & 6920 & 4240 & 5580 & 6480 & 67478.2 \\
\hline 3630 & 3790 & 6820 & 3520 & 4580 & 9850 & 6670 & 5740 & 6130 & 7280 & 67663 \\
\hline
\end{tabular}

\section{CONCLUSION}

In this paper, we introduced a potential type of frauds that we recently discovered in the Smart Grid. Since this type of fraud is a variant of NTL frauds and mutiple fraudsters co-exist or collaborate to commit a fraud, we named them Colluded NTL (CNTL) frauds. We presented our study on the features of this type of frauds, and categorized them into four types: segmented CNTL frauds, fully overlapped CNTL frauds, partially overlapped CNTL frauds, and combined CNTL frauds. We further proposed a detector, named CNFD, to detect CNTL frauds in Smart Grid. We conducted various experiments and the results showed that CNFD can detect four types of CNTL frauds.

\section{ACKNOWLEDGEMENTS}

This work was supported in part by the National Natural Science Foundation of China under the grant 61374200, and the National Science Foundation (NSF) under grant CNS1059265.

The authors would like to thank Dr. Harry Rudin for providing useful comments on presentation and language issues of this paper which significantly enhanced the quality of the paper presentation.

\section{REFERENCES}

[1] J. Gao, Y. Xiao, J. Liu, W. Liang, and C. L. P. Chen. A survey of communication/networking in smart grids. (Elsevier) Future Generation Computer Systems, 28(2):391404, 2012. 


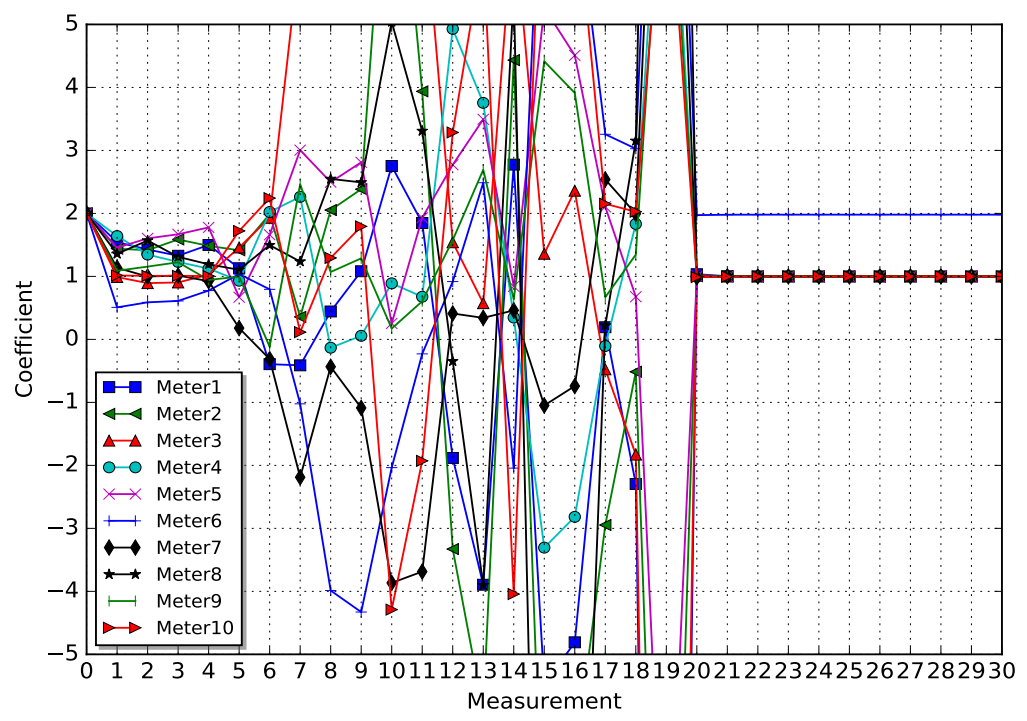

Fig. 10. The result of the first step, tampered meter detection, of CNFD in Exp. 4. It shows the converging process of the coefficients of 10 meters. The coefficient of Meter 6 converges to 1.972 and the coefficients of other meters converge to 1 showing that Meter 6 is the tampered meter.

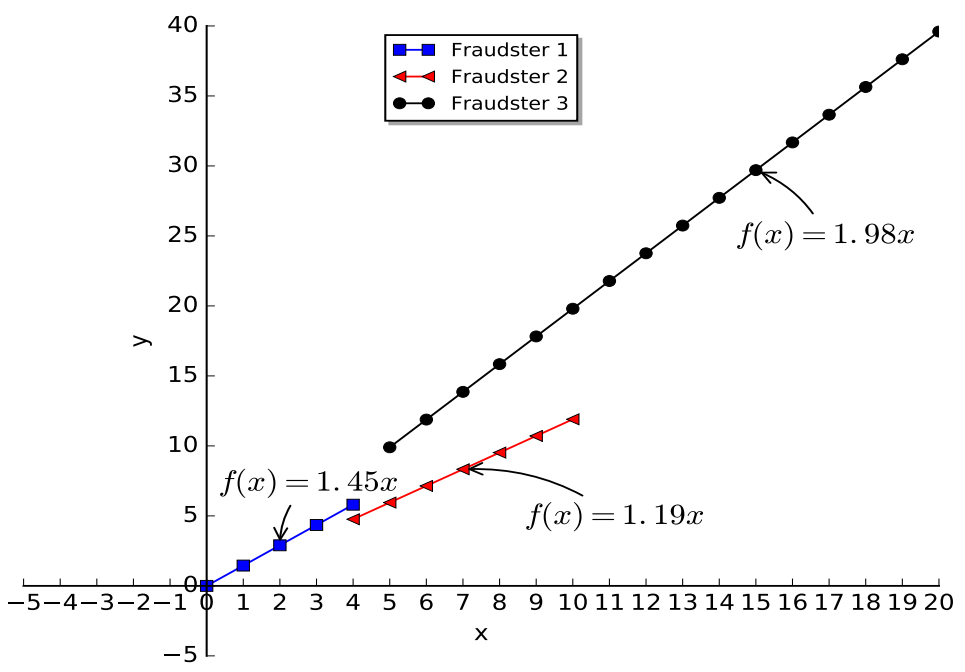

Fig. 11. The result of CNFD after the second step, fraudster differentiation, in Exp. 4. It shows that there is a CNTL fraud on Meter 6 which are committed by three fraudsters.

[2] J. Mu, W. Song, W. Wang, and B. Zhang. Self-healing hierarchical architecture for zigbee network in smart grid application. International Journal of Sensor Networks, 17(2):130-137, 2015.

[3] Z. Xiao, Y. Xiao, and D. Du. Non-repudiation in neighborhood area networks for smart grid. IEEE Communications Magazine, 51(1):1826, Jan. 2013

[4] W. Han and Y. Xiao. Non-technical loss fraud in advanced metering infrastructure in smart grid. In The 2nd International Conference on Cloud Computing and Security (ICCCS 2016), July 2016.

[5] W. Han and Y. Xiao. Combating TNTL: Non-technical loss fraud targeting time-based pricing in smart grid. In The 2nd International Conference on Cloud Computing and Security (ICCCS 2016), July 2016.

[6] Northeast, Group, and LLC. Emerging markets smart grid: Outlook 2015. available at:http://www.northeast-group.com/reports/BrochureEmerging\%20Markets\%20Smart\%20Grid-Outlook\%202015Northeast\%20Group.pdf, Dec. 2014.
[7] P. Detwiler. Electricity theft: A bigger issue than you think. available at:http://www.forbes.com/sites/peterdetwiler/2013/04/23/electricitytheft-a-bigger-issue-than-you-think/\#6a262d5272eff, Apr. 2013.

[8] D. Kundur, X. Feng, S. Mashayekh, S. Liu, T. Zourntos, and K.L. ButlerPurry. Towards modelling the impact of cyber attacks on a smart grid. International Journal of Security and Networks, 6(1):2-13, 2011.

[9] J. Zhang and C. A. Gunter. Application-aware secure multicast for power grid communications. International Journal of Security and Networks, 6(1):40-52, 2011

[10] J. Liu, Y. Xiao, S. Li, W. Liang, and C. L. P. Chen. Cyber security and privacy issues in smart grids. IEEE Communications Surveys \& Tutorials, 14(4):981-997, Fourth Quarter 2012.

[11] Z. Xiao, Y. Xiao, and D. Du. Exploring malicious meter inspection in neighborhood area smart grids. IEEE Transactions on Smart Grid, 4(1):214-226, Mar. 2013.

[12] W. Han and Y. Xiao. Cnfd: A novel scheme to detect colluded non- 
technical loss fraud in smart grid. In Proceedings of The 11th International Conference on Wireless Algorithms, Systems, and Applications (WASA 2016), August 8-10, 2016, Bozeman, Montana, USA., pages 4756, Aug. 2016.

[13] R. Berthier and W. H. Sanders. Monitoring advanced metering infrastructures with amilyzer. Cyber-security of SCADA \& industrial control systems, 2013.

[14] S. McLaughlin, B. Holbert, S. Zonouz, and R. Berthier. AMIDS: A multi-sensor energy theft detection framework for advanced metering infrastructures. In Proceedings of the 2012 IEEE Third International Conference on Smart Grid Communications (SmartGridComm'12), pages 354-359, Nov. 2012.

[15] W. Han, W. Xiong, Y Xiao, M. Ellabidy, A. V. Vasilakos, and N. Xiong. A class of non-statistical traffic anomaly detection in complex network systems. In Proceedings of the 32nd International Conference on Distributed Computing Systems Workshops (ICDCSW'12), pages 640646, June 2012.

[16] Z. A. Baig. Rapid anomaly detection for smart grid infrastructures through hierarchical pattern matching. International Journal of Security and Networks, 7(2):83-94, 2012.

[17] D.J. Gaushell and H.T. Darlington. Supervisory control and data acquisition. Proceedings of the IEEE, 75(3):1645-1658, 2005.

[18] J. Gao, J. Liu, B. Rajan, R. Nori, B. Fu, Y. Xiao, W. Liang, and C. L. P. Chen. Scada communication and security issues. Security and Communication Networks Security Comm, 7(1):175-194, 2014.

[19] P. Rengaraju, S. R. Pandian, and C. Lung. Communication networks and non-technical energy loss control system for smart grid networks. In Proceedings of the 2014 IEEE Innovative Smart Grid Technologies Asia (ISGT ASIA), pages 418-423, May 2014.

[20] J.V. Wijayakulasooriya, D.M.I.S. Dasanayake, P.I. Muthukumarana, H.M.P.P. Kumara, and L.A.D.S.D. Thelisinghe. Remotely accessible single phase energy measuring system. In Proceedings of the 1st Int'l Conf. on Industrial and Information Systems, pages 304-309, Aug. 2006.

[21] A. Pasdar and S. Mirzakuchaki. A solution to remote detecting of illegal electricity usage based on smart metering. In Proceedings of the 2nd Int'l workshop on Soft Computing Applications, pages 163-167, Aug. 2007.

[22] N. Mohammad, A. Baru, and M.A. Arafat. A smart prepaid energy metering system to control electricity theft. In Proceedings of the Int'l Conf. on Power, Energy and Control, pages 562-565, Feb. 2013.

[23] S.S.S.R. Depuru, L. Wang, and V. Devabhaktuni. Support vector machine based data classification for detection of electricity theft. In Proceedings of IEEE/PES Power Systems Conference and Exposition (PSCE), pages 1-8, Mar. 2011.

[24] F. Biscarri, I. Monedero, C. Leon, and J. I. Guerrero. A mining framework to detect non-technical losses in power utilities. In Proceedings of 11th International Conference on Enterprise Information System (ICEIS'09), May 2009.

[25] C. Lin, S. Chen, C. Kuo, and J. Chen. Non-cooperative game model applied to an advanced metering infrastructure for non-technical loss screening in micro-distribution systems. IEEE Transactions on Smart Grid, 5(5):2468-2469, Sept. 2014.

[26] S. Huang, Y. Lo, and C. Lu. Non-technical loss detection using state estimation and analysis of variance. IEEE Transactions on Power Systems, 28(3):2959-2966, Aug. 2013.

[27] X. Xia, W. Liang, Y. Xiao, M. Zheng, and Z. Xiao. Differencecomparison-based approach for malicious meter inspection in neighborhood area smart grids. In Proceedings of the 2015 International Conference on Communications (ICC'15), pages 802-807, June 2015.

[28] X. Xia, W. Liang, Y. Xiao, and M. Zheng. BCGI: A fast approach to detect malicious meters in neighborhood area smart grid. In Proceedings of the 2015 International Conference on Communications (ICC'15), pages 7228-7233, June 2015.

[29] W. Han and Y. Xiao. NFD: A practical scheme to detect non-technical loss fraud in smart grid. In Proceedings of the 2014 International Conference on Communications (ICC'14), pages 605-609, June 2014.

[30] W. Han and Y. Xiao. FNFD: A fast scheme to detect and verify nontechnical loss fraud in smart grid. In Proceedings of the International Workshop on Traffic Measurements for Cybersecurity (WTMC'16), MayJune 2016.

[31] M. H. Hayes. Recursive least squares. In Statistical Digital Signal Processing and Modeling, chapter 9.4, page 154. Wiley, 1996.

[32] W. Han and Y. Xiao. IP ${ }^{2} \mathrm{DM}$ for V2G networks in smart grid. In Proceedings of the 2015 International Conference on Communications (ICC'15), pages 782-787, June 2015.

[33] W. Han and Y. Xiao. Privacy preserving for v2g networks in smart grid: A survey. Computer Communications, 91-92:17-28, 2016.
[34] G. Kalogridis, S.Z. Denic, T. Lewis, and R. Cepeda. Privacy protection system and metrics for hiding electrical events. International Journal of Security and Networks, 6(1):14-27, 2011.

[35] F. Li, B. Luo, and P. Liu. Secure and privacy-preserving information aggregation for smart grids. International Journal of Security and Networks, 6(1):28-39, 2011.

[36] P. Jokar, N. Arianpoo, and V. C. M. Leung. A survey on security issues in smart grids. Security and Communication Networks, 9:262-273, June 2012.

[37] SATEC Power Solutions. Accuracy class: a small $s$ that makes a big difference. available at: http://www.satecglobal.com/sites/default/files/ApplicationNote-Accuracy-ClassDec2012.pdf, 2012.

[38] Georges Habrail and Alice Barard. Individual household electric power consumption data set. available at:https://archive.ics.uci.edu/ml /datasets/Individual+household+electric+power+consumption, 2012.

[39] S. Ma, Y. Yang, Y. Qian, H. Sharif, and M. Alahmad. Energy harvesting for wireless sensor networks: applications and challenges in smart grid. International Journal of Sensor Networks, 21(4):226-241, 2016.

[40] Z. Fu, X. Sun, Q. Liu, L. Zhou, and J. Shu. Achieving efficient cloud search services: Multi-keyword ranked search over encrypted cloud data supporting parallel computing. IEICE Transactions on Communications, E98-B(1):190-200, 2015.

[41] Z. Fu, K. Ren, J. Shu, X. Sun, and F. Huang. Enabling personalized search over encrypted outsourced data with efficiency improvement. IEEE Transactions on Parallel and Distributed Systems, DOI: 10.1109/TPDS.2015.2506573, 2015.

[42] Z. Xia, X. Wang, X. Sun, and Q. Wang. A secure and dynamic multi-keyword ranked search scheme over encrypted cloud data. IEEE Transactions on Parallel and Distributed Systems, 27(2):340-352, 2015.

[43] B. Gu, V. S. Sheng, K. Tay, W. Romano, and S. Li. Incremental support vector learning for ordinal regression. IEEE Transactions on Neural Networks and Learning Systems, 26(7):1403-1416, 2015.

[44] W. Ma, M. Liu, H. Huang, and D. Su. An efficient algorithm for finding frequent and diverse subgraph patterns in ppi networks. International Journal of Sensor Networks, 16(4):210-216, 2014.

[45] B. Gu, X. Sun, and V. S. Sheng. Structural minimax probability machine. IEEE Transactions on Neural Networks and Learning Systems, DOI : 10.1109/TNNLS.2016.2544779, 2016.

[46] Y. Zheng, B. Jeon, D. Xu, Q.M. J. Wu, and H. Zhang. Image segmentation by generalized hierarchical fuzzy c-means algorithm. Journal of Intelligent and Fuzzy Systems, 28(2):961-973, 2015.

[47] H. Yang, S. Fong, K. Cho, and J. Wang. Atmospheric pattern recognition of human activities on ubiquitous sensor network using data stream mining algorithms. International Journal of Sensor Networks, 20(3):147162, 2016.

[48] W. Han and Y. Xiao. Design a fast non-technical loss fraud detector for smart grid. (Wiley Journal of) Security and Communication Networks, accpted. 